\title{
Surgical innovation
}

\section{Saumitra Saha}

Received: 4 February 2007 / Accepted: 19 December 2008

(C) Association of Surgeons of India 2009

\section{Introduction}

Evolution of modern surgery has been a remarkable process. History teaches us that it is impossible to predict what will happen in the future. John Erichsen, a noted English surgeon wrote in 1874 "....the abdomen, chest and brain will forever be closed to operations by a wise and humane surgeon." A few years later Billroth remarked, "A surgeon who tries to suture a heart wound deserves to lose the esteem of his colleagues" [1]. Not only were all these areas subsequently explored with considerable élan, but the most challenging operations have been carried out through the tiniest of incisions and with sophisticated tools. Indeed, minimally invasive procedures have changed the face of surgery. Laparoscopic cholecystectomy had a monumental impact on present day surgery. So did procedures like transurethral resection of prostate, low anterior resection using stapled anastomosis. tension-free mesh repair of hernias and off-pump (beating heart) coronary bypass grafting all with significantly improved outcome. Laparoscopic surgery is being performed for a host of other abdominal conditions and is beginning to quell fears of oncological inadequacy, particularly in colorectal cancer. There have been innumerable other 'newer' procedures in surgery but not all have had the desired impact. There is a need for critical analysis if technological break-through and surgical innovation is to be understood in the context of ever-changing surgical practice.

\section{What is innovation?}

Innovative therapy is characterized by being both novel and non-validated. It encompasses significant modification of a standard technique, a new application of or a new indication for an established technique, or an alternative combination of an established technique with another therapeutic modality that was developed and tested for the first time [2]. 
The Belmont Commission report in 1978 suggested that innovations should be incorporated into a research project [3]. However, there have been innovations which were perceived to have such profound benefits that they were introduced and widely disseminated without proper evaluation by clinical trials, most notably laparoscopic cholecystectomy, organ transplantation and joint replacements. This is not surprising since only about $20 \%$ of surgical techniques have undergone clinical trials in the last four decades [4]. Most surgeons have spontaneously innovated at some point or other while operating in less than optimal conditions or when faced with a difficult problem which only requires the safeguard of an individual or departmental audit. It is the more deliberately planned changes that have wider ramifications. The modifications of commonly accepted procedures may be in small incremental steps and the impact of such innovations depends on the extent to which novelty is claimed. While the aim of any new procedure is to maximize benefits to the patient, it must also be realized that the uncertainties associated with innovations may increase the risk of complications.

\section{Innovation vis-à-vis technical refinement and experimentation}

Uncertainties and disagreement exist as to what is an acceptable variation on an existing technique and also when does the introduction of an innovation amounts to human experimentation warranting a rigorous trial. An innovation is significant only when it has stood the test of time and clinical trials. The history of surgery is replete with examples of operations that were developed without the rigours of clinical trials, only later to be shown as ineffective or even harmful. Prefrontal lobotomy for schizophrenia, extended radical mastectomy for breast cancer, jejuno-ileal bypass for obesity, cervical rib resection for scalenus anticus syndrome, extracranial-intracranial (EC-IC) bypass to prevent recurrence of stroke and more recently arthroscopic knee surgery for osteoarthritis are some of the well known examples [5]. In the quest for technological advancements, innovations can become unregulated and may not bring about the desired outcome which matters most to the patient. One might do an operation in a certain way and believe that it is technically superior compared to an earlier procedure, but does not have evidence that the modification is a genuine improvement. There has often been a gradual shift in operative technique for a given problem, such that it is a question of degree as to when a modification becomes an innovation. The evolution of open surgery for primary inguinal hernia best exemplifies it. The Lichtenstein technique of tension-free mesh repair was a significant innovation as it radically changed the approach to hernia repairs with excellent results. A host of new "mesh operations" followed, varying the shape and size of mesh and placement techniques, which are essentially technical modifications and do not have the same ingenuity as the Lichtenstein technique. They include Mesh plug repair, Perfix plug ${ }^{\circledR}$ and patch repair, Prolene Hernia System ${ }^{\circledR}$ repair, sutureless preshaped mesh repair (Trabucco), sutureless repair with umbrella plug (Gilbert), dynamic self regulating prosthesis repair (Valenti), 4DDOME ${ }^{\circledR}$ repair and self expanding preperitoneal mesh repair (Kugel). Also, none of these have significantly improved on the recurrence rate (the principal outcome measure) of the original Lichtenstein operation although they may have altered some surrogate endpoints (indirect outcomes) or are applicable in special situations. The flurry of hernia repair techniques have created confusion, even amongst hernia surgeons prompting them to deliberate on the theme of "Anarchy in Hernia Surgery" in a recent World Congress in Naples, Italy [6]. Like operations, surgical tools also evolve e.g., efforts are under way to make laparoscopic instruments which would give tactile feedback to surgeons [7]. This is obviously a refinement of the existing apparatus and not a novelty like the radiofrequency ablation treatment for liver tumours [8]. Transurethral resection of prostate (TURP) brought a paradigm shift in the management of benign prostatic hyperplasia. The more recent variations like transurethral microwave thermotherapy, needle ablation and laser vaporization although employing the same route has further minimized the trauma and allows outpatient treatment even in high risk elderly patients. These procedures deserve to be labeled as innovations as a new concept of ablation rather than resection was successfully put into practice.

\section{Role of market forces}

There is always a marketing advantage for a less invasive procedure - it appeals to both the patient and the surgeon and this alone may cloud the real outcomes of an operation such as survival, recurrence rates and major complications. Often an innovation is translated into a huge commercial exercise, pushing the cost of surgery up. Conversely, costly changes are more likely to be considered novel and better, whereas less costly changes may not get widespread attention or is met with contempt. Time will tell whether Desarda's ingenious concept of physiological and dynamic posterior wall repair with external oblique aponeurosis which is considerably cheaper than commercial mesh repair would emerge as a significant innovation, particularly in resource-poor countries [9]. The consumer marketing strategy that a costly innovation ought to be better than the existing technique has led to a spiraling healthcare costs and has prompted medical insurance companies to adopt stringent criteria for payouts. The recent National Emphysema Treatment Trial (NETT) for chronic obstructive airway disease comparing medical treatment to "lung volume reduction surgery" was supported by American insurance companies, 
and set a precedent by making involvement in a randomized controlled trial a condition for reimbursement of the costs of the new operation [10]. Economic factors determine whether an innovation would be widely adopted or not. Despite being a technological marvel, the benefits of the da Vinci Surgical System (an operative platform for robotic surgery) may be confined to the costliest health systems in the world, due to the astronomical cost of installation, maintenance and training. Similarly, the concept of Natural Orifice Transluminal Endoscopic Surgery (NOTES) for carrying out cholecystectomy, appendicectomy, splenectomy etc has been questioned, given the advanced state of laparoscopic surgery today. Will there be a true clinical advantage to avoiding incisions in the abdominal wall? Can the visceral (stomach) wound be closed safely and reliably? Will the gastroenterologists and surgeons from contrasting backgrounds and training get together to make this a truly trans-disciplinary procedure? Whether the enormous funding and enthusiasm that has gone into development of NOTES tools is able to overcome such real concerns and save this procedure from becoming a 'surgical footnote' may not be clear for some years [11].

\section{Ethical issues and regulation of innovation}

A new concept stimulates a profusion of interventions and technological modifications and it is this aspect along with the associated commercial interests that require regulation. The aim should not be to stifle originality but to introduce changes that are unequivocally beneficial into surgical practice. Efforts have been made to evaluate new interventional procedures by regulatory boards such as the Interventional Procedures Advisory Board of the National Institute of Clinical Excellence (NICE) in the UK. They recommend whether a new procedure is suitable for normal use, special use (with mandatory clinical governance), other use (taking into account training and service provisions), research or unsuitable in clinical practice. The driving force behind today's innovation is technology and is usually an evolutionary process rather than a sporadic revolutionary incident [12]. That, innovation however novel they are should be a part of research protocol and performed with special consent highlighting the uncertainties have been the message from NICE with procedures like Totally Endoscopic robotically assisted Coronary Artery Bypass grafting (TECAB) [13]. Similar technological assessments are also performed by The Australian Safety and Efficacy Register of New Interventional Procedures- Surgical (ASERNIP-S) who have allowed the general public and medical indemnity organizations besides health professionals and hospital credentialing committees to notify and nominate new interventions for a formal 'safety and efficacy' assessment. There is every possibility that in future, surgeons performing newer/ experimental procedures might not be covered by medical indemnity until they carry out the procedure within the framework of regulatory guidelines. Implicit in many of these guidelines are the fact that an innovative technique might show improved results once the learning phase is over. A look at the list of procedures nominated to ASERNIP-S for evaluation reveals wide-ranging procedures such as spinal endoscopy, radiofrequency ablation of tumours other than in liver, endovascular intracranial aneurysm surgery, colonoscopic stents and small vessel angioplasty [14].

The principal ethical issue associated with surgical innovation is the element of unknown risks which comes with any novel procedure and the harm that one might cause during the learning phase of a new operation. This may be at odds to the principle of "First, do no harm" enshrined in our surgical ethos but if we do not take risks, we will never reap the benefits of innovation. While the checks outlined in Table 1 may act as a deterrent to indiscriminate experimentation it is the individual surgeon's belief that stimulates surgical innovation. It is hard to commit to a new procedure with its attendant risks, if one does not believe that there is going to be a significant benefit.

\section{Summary}

Surgical innovations are inevitable but not all would be subjected to trials in the same way as a new drug, because of the difficulty in establishing equipoise (genuine uncertainty about relative merits of different approaches) and the skill-dependence of surgery. The needs of rural areas are also different as opposed to those perceived by surgeons

Table 1 Multi-level checks to reduce risks associated with surgical innovations

\begin{tabular}{l}
\hline Individual (Surgeon) \\
Adequate training \\
Assessment (independent of claims by the manufacturer) \\
of the evidence of effectiveness and safety of a new \\
device \\
Peer-review and guidance \\
Informed consent \\
Institutions / Hospitals \\
Clinical Audit (Outcome) \\
Health related quality of life (HRQOL) assessment \\
Periodic validation \\
Cost-effectiveness study \\
Professional bodies \\
Impart appropriate training \\
Accreditation for newer procedures \\
Disseminate evidence from rigorous RCTs \\
Formulate national guidelines \\
Create national registry of innovative procedures \\
Government and other regulatory bodies (in conjunction with \\
Professional bodies) \\
Set-up standards of evidence required for licensing new \\
surgical devices \\
Appropriate patenting procedures
\end{tabular}


working under ideal conditions in developed countries. Purely from the health economics point of view, the use of a cheap mesh made from autoclavable mosquito-net cloth (with a cost ratio of 1:2000) by rural surgeons, although considered a surgical blasphemy by many, could assume a significant position in hernia surgery of many countries including India $[15,16]$. Human nature, more so that of surgeons tend to resist change but there is also a paradoxical eagerness to embrace innovative technology. This could be due to external pressures such as the clamour of the public and manufacturers to introduce a new technique rapidly. The media by glorifying and sensationalizing new technology that are yet to be validated have also played its part in it. Such influences do not apply, for example, in changing the age-old practice of withholding feeds for prolonged periods after gastrointestinal surgery to the more scientific early postoperative enteral feeding. Innovations should intend to improve outcomes that really matter and be guided by a genuine clinical need and not by the "idols of the marketplace or the vagaries of clinical opinion" [17]. The concept of 'Health Technology Assessment' is largely non-existent in India but the increasing globalization, rising costs, litigation and health insurance will inevitably influence the way we embrace surgical innovations.

Conflict of interest The author do not have any

disclosable interest

\section{References}

1. Rutkow IM (1993) Surgery: An illustrated history. St Louis: Mosby

2. Reitsma AM, Moreno JD (2002) Ethical regulations for innovative surgery: the last frontier? J Am Coll Surg 194: 792-801

3. McCarthy CR (1984) Regulatory aspects of the distinction between research and medical practice. IRB 6:7-8
4. Brower V (2003) The ethics of innovation. EMBO reports 4:338-340

5. Morgenstern L (2006) Innovative Surgery's Dilemma. Surgical Innovation 13:73-74

6. Corcione F (2005) Preface In Corcione F (editor) New Procedures in Open Hernia Surgery. New Delhi: Springer

7. The BUPA Foundation, London. Available at www.bupafoundation.com/asp/about/latest_news_artcles/ surgical innovation.asp. Accessed on 2nd February 2007

8. Lin SM, Lin CJ, Lin CC, Hsu CW, Chen YC (2005) Randomised controlled trial comparing percutaneous radiofrequency thermal ablation, percutaneous ethanol injection, and percutaneous acetic acid injection to treat hepatocellular carcinoma of $3 \mathrm{~cm}$ or less. Gut 54:11511156

9. Desarda MP (2006) Physiological repair of inguinal hernia: a new technique (study of 860 patients). Hernia 10: 143-146

10. Carino T, Scheingold S, Tunis S (2004) Using clinical trials as a condition of coverage. Clin Trials 1:108-121

11. Baron TH (2007) Natural orifice transluminal endoscopic surgery. Br J Surg 94:1-2

12. Iserson KV, Chiasson PM (2002) The ethics of applying new medical technologies. Semin Laparosc Surg 9:222-229

13. National Institute of Clinical Excellence. Totally endoscopic robotically assisted coronary artery bypass grafting. Interventional procedure guidance 128. NICE: London, 2005

14. Australian Safety and Efficacy Register of New Interventional Procedures- Surgical, Sydney. Available at www.surgeons.org/Content/NavigationMenu. Accessed on 2nd February 2007

15. Udwadia TE (2006) Inguinal hernia repair: The total picture. Journal of Minimal Access Surgery 2:144-146

16. Tongaonkar RR, Reddy BV, Mehta VK, Singh NS, Shivada S (2003) Preliminary multicentric trial of cheap indigenous mosquito-net cloth for tension-free hernia repair. Ind J Surg 65:89-95

17. Marks HM (1997) The progress of experiment. New York: Cambridge University Press 